\title{
Analysis of Applied Mathematics in Big Data Era
}

\author{
Shi Donhe \\ Xi' an International University, Business School 710077, China
}

Keywords: analysis, applied mathematics, big data era

\begin{abstract}
By introducing the current state of application of applied mathematics, combined with the background of the era of big data, analysis in the era of big data, while the requirements for data analysis continue to increase, at the same time, the methodological requirements for applied mathematics are also increasing, which has led to the demand for professional applications grows. Combined with management analysis methods, the application of mathematical mathematics is analyzed in the era of big data. It is concluded that in the era of big data, the application prospects of applied mathematics will greatly improve.
\end{abstract}

\section{Introduction}

With the popularity of the Internet and various smart devices, various types of data have experienced explosive growth, and technologies such as cloud storage and cloud computing are helping us to store vast amounts of information and dig out what people need from these information, so our time has been Called the era of big data. Big data technology requires the freedom, openness and sharing of data. We have entered the era of data sharing, but we are also constantly exposed to the "third eye". Therefore, big data technology brings about the hidden dangers of personal privacy protection. It also brings about the fear of abuse or monopoly of data by individual organizations. In particular, human sacred free will may be infringed, resulting in the era of big data. The issue of freedom and responsibility has brought new challenges to the traditional ethics [1].

\section{Related Research in the Era of Big Data}

We think that the definition of big data is divergent, mainly because the content of big data is too "big". Everyone sees it from different angles, and then there is a situation where benevolent people see the wisdom and the wise see wisdom. According to the historical evolution of big data and the era background of big data, we can fully understand the meaning of big data.

In the era of big data, data leads people's lives and guides business transformation and technological innovation. From the perspective of the era of big data, we can regard big data as the research object and understand big data from both the data itself and the technology of processing data. This understanding of big data has a narrow and broad sense: The narrow sense of big data is the structure and scale of the data is understood from the literal meaning of the data. Broad data in broad sense includes not only the structure of the data and the size of the data, but also the technology of processing the data.

Big data technology is a new technology revolution that is currently emerging. It is essentially a continuation of the information revolution. It makes Thorne's third wave of ideas or the post-industrial society depicted by Daniel Bell really turn into reality. . Data is a well-known digital code, that is, digital coding plus its background knowledge. It is a scientific expression of information. The so-called big data, literally speaking, means that the data volume in the database is particularly large, and it has reached the so-called PB or ZB byte even larger [2]. However, the large data capacity is only a superficial phenomenon. The real essence of big data is its data worldview and thinking mode. In the big data world, everything in the world can be quantified, and the entire world is a data world. According to the way of thinking of big data, we should analyze any problems with a holistic view of data and analyze them with correlation analysis [3]. 
The revolution in big data technology is facilitated by the rapid development of various scientific technologies such as computer technology, intelligent technology, network technology, and cloud technology. Data descriptions and analyses of things have long existed. Ancient civilizations such as ancient Egypt, ancient Greece, and ancient China have long used data to record assets, calculate wealth, count populations, collect taxes, and so on. The modern science after the Renaissance was the digitization of the natural world and the use of mathematics and computational methods to grasp the laws of the natural world, bringing about a revolution in modern science and technology. With the rise of big data technology, areas that were previously difficult to digitize, such as human spirit and behavior, can all be characterized by data. Therefore, the field of data is continuously expanding, and the vision of quantifying everything has basically been realized. 2012 was known as the world's first year of big data, and 2013 was known as China's first year of big data. This marked the rapid progress of human society into the era of big data.

Compared with traditional data technologies, with the support of advanced technologies such as smart technologies, network technologies, cloud storage, cloud computing, and the Internet of Things, big data technology has the following four characteristics: Large quantities, ie extremely large data scale; The type is miscellaneous, namely the data source is diversified, the structure type is complex; The speed is quick, the data acquisition, the transmission and the processing may realize the real-time on-line; 4th, the value is high, namely the commercial value of the data is high, but the value density is low.

Big data technology not only brought about a technological revolution in data collection, storage, transfer, and use, but also triggered a tremendous change in people's ideas and brought the spirit of "freedom, openness, and sharing" in the era of big data. In the era of small data, due to limited data resources, people generally store relevant data as secrets, such as laboratory scientific experimental data, personal consumption data of daily life, and so on. People are all in their own right and do not want others to know their whereabouts and thoughts. Similarly, we cannot know other people's data. But in the era of big data, we live in the vast ocean of data and all kinds of data are stored in the network, stored in the cloud, so it is a completely open data ocean. People in the ocean of data are free to dig and get the data they need. In the era of big data, people are more willing to expose themselves to the Internet and share it with people. Therefore, "freedom, openness, and sharing" became a common declaration in the era of big data [4].

\section{The Development of Applied Mathematics}

Applying mathematics as a discipline derived from mathematics, the development of the composite subject has become more and more important. For example, the Mathematics Academy of Peking University has opened information science, computer science, statistics, and financial mathematics as applied mathematics, and these directions are often sought after by the industry and academic circles.

Applied mathematics is different from pure mathematics. It only studies mathematics theory but contains two aspects. The first is related to the application of mathematics, the second is the application of mathematics, that is, mathematics as a tool to solve the problem, to explore the issues of engineering and humanities and social sciences, and to carry out cross-disciplinary research. The direction is mainly applied in two areas. The first is the computer field. This includes the traditional direction of information science and the emerging direction of data science (big data and artificial intelligence). With the rapid advancement of computer technology, in addition to the computer itself the technological innovation requires basic theories such as mathematics to provide theoretical basis, but also requires the use of mathematical mathematics to provide algorithm optimization support during the data analysis and other phases. This highlights that the computer science has been inseparable from the support of applied mathematics. The second is the field of financial economics. The study of modern economics is divided into theoretical and empirical studies. When economists have completed a theoretical study or an economic model, they need to use data for empirical research, such as the measurement of economics in economics. Learning is to solve practical economic problems on the basis of the combination of traditional economic theory and applied 
mathematics knowledge. In recent years, under the background of the increasing importance of quantitative economics, the combination of economics and mathematics will be more in-depth and extensive, with unlimited prospects.

In addition to the application of applied mathematics in the research fields of some composite disciplines, it can also be observed from the perspective of college curriculum. As one of the top business schools, Peking University's HSBC School of Business, in the enrollment phase of its Master of Finance program, specializes in setting up quantitative financial masters for mathematics students, which is different from other major financial enrollment students, and the fiercest competition. In addition, according to the "Daily Economic News" report, undergraduate entrance examinations, undergraduate students from applied mathematics, when they apply for master's degree in the selection direction has a great advantage, you can turn to big data, finance, computer masters and other biased applications The direction, and this type of student is very much sought after by various colleges and universities. Above, from a side, it reflects the wide range of applications of applied mathematics and the prospects are good.

\section{The Applications and Prospects of Applied Mathematics in the Era of Big Data}

Applied mathematics aims to teach students the basic theory and basic methods of mathematics science, develop their ability to use mathematical knowledge to use computer technology to solve practical problems, and provide research, teaching or production for social sciences, education and economic sectors. High-level talents such as business applications, development research, and management work, while Big Data is a collection of data that cannot be captured, managed, and processed using conventional software tools within an affordable timeframe. Professionals are computer professionals. In big data processing applications, more knowledge of applied mathematics will be incorporated to achieve further application of applied mathematics in big data processing. As a basic discipline, there is a great need for funds in the field of mathematics, and it is difficult for the invested gold to obtain substantial economic returns in the short term. Benefiting from the research results of the previous generation, most of the current mathematical theories are relatively complete, but for mathematicians, the road to making more academic breakthroughs on this basis seems thorny. At present, China's investment in mathematics research is limited. At the same time, basic mathematics and applied mathematics have started late, and their development is still at a disadvantage internationally. Compared with mathematics, although the big data field started at an early stage but developed rapidly, the industry's output value has increased year by year, and the application market has become increasingly broad. The enormous economic value it contains is not to be underestimated.

Mathematics and big data have developed together and can now be demonstrated economically and technologically. Fully tapping the economic potential of big data, the huge output value created by big data can be used as capital to invest in mathematics and promote the in-depth development of mathematics research; mathematics academia can make full and flexible use of funds to maximize and enrich existing mathematics theory. For example, the use of funds to hold international mathematics seminars will create conditions for the collision of mathematicians' thought sparks. This will also help narrow the gap between domestic mathematics research and the international top level and promote the academic prosperity of mathematics. The improvement of basic mathematics theory and the ease of application of mathematics will also be used as a technical prerequisite to promote greater prosperity in the field of big data. Both sides will promote each other, develop together, and develop together for mutual benefit [5].

In the future, the common development of mathematics and big data may be expressed at the theoretical level. The data excavated by big data is comprehensive and trivial, and the conclusions are light and concise. However, the relevant conclusions concluded through objective data are of great value. They can provide reference for mathematicians and are expected to explore new mathematical laws.

Under the background of the rapid development of the big data industry, the combination of big data and financial, artificial intelligence, and computer industries has become more and more 
in-depth, and the development of pure mathematics and applied mathematics has also received widespread attention from the society, especially applied mathematics. However, as a basic discipline with high input, research funding for mathematics is subject to certain restrictions. With the deep integration of big data and other industries, and the rapid increase in output value, Big Data will increase the demand for mathematical research and at the same time the benefits of its data industry. It will also bring more research funding for mathematics research, so as to further promote the in-depth study of mathematics, and then consider that in the past two years, mathematics + big data composite talents have been sought after by companies. Therefore, the prospects for the development of math + big data in the future will steadily increase.

At present, although the big data industry has received strong support from the state and the corporate level, it has to admit that it is still in a period of growth and has more room for development. Therefore, the mathematics major should grasp this development opportunity, combine more mathematical theory with big data knowledge, take the initiative, and take the initiative. In particular, in terms of personnel training, the mathematics major should pay more attention to the cultivation of compound talents so that high-end talents can master both mathematics theory and computer practice, and apply advanced mathematics theory to the upgrading of the big data industry. This will truly promote mathematics. The prospect of professional application is really expanded.

With the increasing attention of applied mathematics, the pure mathematics research that is the foundation of the discipline development has been neglected, which will also become a challenge to mathematics applications. If we place too much emphasis on the application of mathematics, we are eager to transform mathematical achievements into productivity. To a certain extent, it is against the law of development of mathematics. After all, mathematics is still a basic discipline, so it requires a long period of solid research before it is possible to achieve impressive results. In the era of big data, the close integration of mathematical applications and data processing will make the basic subject of mathematics more utilitarian, which will cast a shadow on the study of pure mathematics. There is no foundation for rich basic mathematics research.

\section{Conclusion}

In the era of big data, the increasing demand for algorithm optimization will drive the rapid growth in the application of applied mathematics. If applied mathematics can do cross-disciplines, applied mathematics theory can be better applied to mathematical models related to data processing, and play a role in methodology and then the application of applied mathematics will be widely used.

\section{References}

[1] Zhu Jianping, Zhang Guijun, Liu Xiaotong. Differentiation of data analysis ideas in the era of big data [J]. Statistical Research, 2014(2): 10 - 17.

[2] Li Jianzhong, Liu Xianmin. An important aspect of big data: Data availability [J]. Computer Research and Development, 2013, 50(6): 1147 - 1162.

[3] Li Guojie, Cheng Xueqi. Big Data Research: The major strategic areas for future science and technology and economic and social development [J]. Proceedings of the Chinese Academy of Sciences, 2012, 27(6): 647 - 657.

[4] Feng Dengguo, Zhang Min, Li Yan. Big Data Security and Privacy Protection [J]. Chinese Journal of Computers, 2014, 37(1): 246 - 258.

[5] Meng Xiaofeng, kindness. Big Data Management: Concepts, Techniques, and Challenges [J]. Computer Research and Development, 2013, 50(1): 146 - 169. 